\title{
Inseminación artificial en la especie porcina: dosis inseminante en relación con el lugar de deposición
}

\author{
Artificial insemination in the swine species: inseminating dose related to \\ the deposition place
}

\author{
Compagnoni Maricel Vanina ${ }^{1,3}{ }^{*}$ (i) , TitTarelli Claudia Marcela ${ }^{1}$, (i) \\ WILLIAMS SARA INÉS ${ }^{1,2}$ (1)
}

1. Cátedra de Reproducción Animal, Facultad de Ciencias Veterinarias, Universidad Nacional de La Plata (UNLP), Argentina. 2. Cátedra de Producción Porcina. Facultad de Ciencias Veterinarias, Universidad Nacional de La Plata (UNLP), Argentina. 3. Consejo Nacional de Investigaciones Científicas y Técnicas (CONICET-CCT-La Plata), Argentina.

* Correo electrónico de la autora de contacto: mcomapagnoni@fcv.unlp.edu.ar

\begin{abstract}
Resumen
La inseminación artificial en la especie porcina es una herramienta de gran utilidad que permite grandes avances en la industria porcícola, tanto en nuestro país como en el mundo. La inseminación artificial es una herramienta biotecnológica de la reproducción que se aplica en la producción animal con el fin de aumentar la eficiencia productiva, lograr un mayor progreso genético e incrementar el desempeño reproductivo. Existen diferentes métodos de inseminación artificial en porcinos que permiten variar el volumen y la cantidad de espermatozoides de la dosis inseminante al modificar el lugar de deposición de esta. El objetivo de esta revisión fue recopilar y actualizar información relevante sobre la inseminación artificial en la especie porcina, relacionando la dosis seminal y el método de conservación con la técnica empleada.
\end{abstract}

\section{Palabras clave}

Porcino, semen, inseminación artificial, dosis inseminante

\begin{abstract}
Artificial insemination is a very useful tool that allows huge advances in the swine industry all around the world. The use of artificial insemination for breeding pigs has facilitated global improvements in genetic and reproductive performances. There are variations of the technique with differences in the volume and number of sperm per dose according to the place of deposition of the semen. This review is a compilation and update of relevant information on artificial insemination in swine, relating the seminal dose and the preservation method with the technique used.
\end{abstract}

\section{Key words}

Swine, sperm, artificial insemination, inseminating dose

Fecha de recepción: 17/04/2019

Fecha de revisión: 30/09/2019

Fecha de aprobación: 11/10/2019
ANALECTA Vet 2019; Julio-Diciembre; 39(2):33-46

Impresa ISSN 03655 14-8 Electrónica ISSN 1514-2590

doi.org/10.24215/15142590e041 


\section{Introducción}

La biotecnología ofrece técnicas que aplicadas a la reproducción animal aumentan la eficiencia productiva en diferentes especies domésticas. Su objetivo es lograr un mayor progreso genético para incrementar la producción de leche, lana, pelo y carne, según la especie en cuestión (Palma, 2001). Un ejemplo de ellas es la inseminación artificial (IA), método de reproducción asistida que consiste en colocar espermatozoides viables en el tracto reproductivo de una hembra en celo, con el objetivo de lograr una preñez exitosa. De manera más extensa podemos definir a la IA como la técnica mediante la cual es posible extraer semen a un reproductor, diluirlo y conservarlo, con el propósito de llevarlo al lugar ideal del aparato genital de la hembra, a fin de fecundarla, realizando esta técnica en el momento oportuno y con el instrumental adecuado (Rillo, 1982). La mejor forma de aprovechar el potencial genético de un verraco es la IA, por el efecto multiplicador que la misma ejerce sobre el semen. Además, facilita la implementación de programas de cruzamiento, mejores controles sanitarios, de bioseguridad y productivos (Rillo, 1982). Actualmente, se utiliza como herramienta para aplicar otras nuevas técnicas de la reproducción, como son el sexado de espermatozoides, la criopreservación de semen y otros métodos mucho más complejos, como la transgénesis (Palma, 2001).

Uno de los objetivos primordiales de la IA es incrementar el recurso macho; para ello, luego de la extracción de semen, este se diluye para obtener dosis seminales que contengan una cantidad de espermatozoides apropiada según los métodos de conservación y de IA que se utilizarán. Es así como el semen de machos de alto mérito genético puede ser utilizado para inseminar de 10 a 20 cerdas partiendo de un único eyaculado (Rillo, 1982). Como se podrá deducir de lo expresado anteriormente, la cantidad de espermatozoides por dosis modificará de forma directa el rendimiento del eyaculado.

Es de suma importancia determinar el momento ideal para realizar la IA. Esto es debido a que, en la especie porcina, no solo es importante conseguir la preñez, sino, además, obtener la mayor cantidad de embriones posible en cada gestación, para lograr un tamaño de camada adecuado a las exigencias productivas de cada establecimiento comercial.

La IA en la especie porcina ha sido un instrumento de gran utilidad para la mejora global de las piaras en cuanto a fertilidad, genética, sanidad y manejo. Los primeros avances logrados en conservación de semen permitieron extender la vida útil de las dosis seminales hasta por 3 días, lo que posibilitó su envío a distancias relativamente próximas al lugar donde se encontraban los machos. Con los progresos en el desarrollo de diluyentes de larga duración, la capacidad de conservar semen durante un período más prolongado abrió las puertas a una rápida aceptación de la IA en todo el mundo (Knox, 2016).

En la actualidad, las granjas comerciales utilizan la IA para realizar los servicios. Los mismos se efectúan en el momento que se detecta el celo, recibiendo cada hembra al menos dos dosis de semen por ciclo. Esta práctica permite reducir el porcentaje de machos en el establecimiento al 1$2 \%$ (Franco, 2012).

Los últimos avances realizados para incrementar la mejora genética a través del uso de la IA se centraron en reducir la cantidad de espermatozoides por dosis y la cantidad de IA por celo, con el objetivo de lograr una preñez exitosa luego de realizar una única IA posterior a la inducción de la ovulación (inseminación artificial a tiempo fijo). Ambos enfoques permiten una mayor presión de selección para caracteres económicamente importantes desde el punto de vista productivo, y ayudan a extender las ventajas genéticas a través de la IA a más granjas de producción (Knox, 2016).

El objetivo de esta revisión fue recopilar y actualizar información relevante sobre la IA en la especie porcina, relacionando la dosis seminal y método de conservación, con la técnica empleada.

\subsection{La inseminación artificial porcina en el mundo}

Los inicios de la técnica de IA en porcinos ocurrieron en los países desarrollados, y su difusión global hizo que llegara a nuestro país para ser adoptada por los productores locales. Las primeras contribuciones para el desarrollo de esta técnica revolucionaria en la especie se realizaron entre 1926 y 1940 en Rusia, Estados Unidos, Japón y Europa. Hacia la década del '6o la monta natural comenzó a ser reemplazada por la IA. Su aplicación fue reportada por primera vez en el Reino Unido, algunos países de Europa (Brassley, 2007) y otros lugares del mundo (Niwa, 1961, citado por Knox, 2016).

Ya para mediados de la década del ' 80 , algunos países de Europa practicaban IA en más del 50\% del plantel de reproductoras, mientras que, en otros países, la misma se fue incorporando de manera gradual (Crabo, 1991). Asimismo, en esta década, se constituyeron los primeros centros de IA, en los que una cantidad significativa de verracos fueron confinados con el objetivo de producir semen (Reed, 1982).

En muchos países de Europa, durante los años '9o, los registros indicaban que la mayoría de los cerdos destinados a consumo eran producto del uso de la IA. En el año 2000, el incremento de la utilización de la IA en el mundo llevó a que prácticamente todos los cerdos fueran producidos mediante esta técnica (Weitze, 2000). Su incorporación en muchos países en desarrollo también ocurrió a pesar de las limitaciones de infraestructura. 
La eficiencia y las ganancias de las producciones comerciales están ligadas al uso de la IA. Los registros reproductivos de cientos de miles de cerdas mostraron, ya en 1990, que las tasas de partos logradas con el uso de IA variaban entre el 80 y el $90 \%$ y que el tamaño de las camadas obtenidas era de 11 a 13 lechones (Colenbrander et al., 1993; PigCHAMP Benchmarking, 2012). Más recientemente, en el año 2014, datos provenientes de un relevamiento de 1,3 millones de cerdas, mostraron una tasa de parto del $86 \%$, con un promedio de 14 lechones nacidos totales (Yeste et al., 2014). A pesar de esto, el nivel de fertilidad alcanzado es muy variable entre países y granjas y puede atribuirse a diferencias en los métodos de trabajo a la hora de producir las dosis seminales y efectuar la IA propiamente dicha.

\subsection{La inseminación artificial porcina en la República Argentina}

En Argentina, el uso de la IA en la especie porcina se inició en 1953, cuando se creía que su aplicación era de utilidad solo con fines científicos (Cano \& García Mata, 1953). No fue sino hasta la década del '70 que algunos profesionales del ámbito privado hicieron esfuerzos aislados para implementar esta técnica en establecimientos comerciales. Si bien para esta época la inseminación en bovinos era ampliamente conocida y su empleo se hacía cada vez más creciente, no ocurría lo mismo en el sector porcino (Arisnabarreta \& Allende, 2017). La falta de difusión entre los productores porcícolas pudo deberse al escaso interés de estos por intentar una mejora genética de sus planteles. Otros inconvenientes con los que debían lidiar, era la imposibilidad de conservar el material seminal durante tiempos prolongados y la incertidumbre de cuál sería el momento óptimo para realizar la inseminación, debido a la relativamente larga duración del celo (Arisnabarreta \& Allende, 2017).

Marotta (1973; 1978), docente y difusor de la IA porcina desde una perspectiva académica, publicó en la Revista de la Sociedad de Medicina Veterinaria, dos artículos detallando, por un lado, las características del semen y la eyaculación (extracción, conservación y dilución del material seminal) y, por el otro, el tiempo de conservación y la técnica de inseminación. Sin embargo, en lo que respecta a su aplicación, solo mencionó a los grandes productores mundiales de carne de cerdo (Japón, Europa y Estados Unidos) como países que la utilizaban.

Hacia la década del '80 se establecieron los primeros dos centros de IA porcina. En estas instalaciones se pretendía recolectar, procesar, acondicionar y enviar a distancia el material seminal de verracos entrenados para dicho fin. Uno de ellos fue el Centro de IA porcina de Inriville (Córdoba), con instalaciones modelo, diseñadas bajo la supervisión técnica del INRA (Instituto Nacional de Investigación Agronómica de Francia) aunque, por diferentes razones, este centro nunca llegó a funcionar como tal. El segundo, fue el Centro de IA porcino CAR-CIAVT que funcionó en el Centro Agrotécnico Regional de Venado Tuerto (Santa $\mathrm{Fe}$ ), bajo la dirección técnica del CIAVT (Centro de Inseminación Artificial de Venado Tuerto). Uno de sus objetivos fue capacitar a los potenciales usuarios, productores y profesionales, en el manejo de la técnica de la IA porcina. El CAR-CIAVT trabajó durante cinco años, con diez verracos de diferentes razas, la mitad de los cuales se importaron de Canadá y Estados Unidos. Si bien desde el punto de vista técnico este proyecto fue un éxito, no logró alcanzar las expectativas comerciales esperadas, debido a que solo pudieron comercializar 2400 dosis/año. En consecuencia, se decidió devolver los verracos a sus propietarios y capacitar al personal para que realizara la obtención y dilución del semen en sus propios establecimientos comerciales (Arisnabarreta \& Allende, 2017), modalidad que actualmente se sigue poniendo en práctica.

Según Arisnabarreta y Allende (2017), durante los últimos años de la década del '90 en Argentina, la aplicación de la IA porcina se difundió ampliamente, impulsada por el desarrollo de programas de mejoramiento genético. Coincidentemente, Riesenbeck (2011), menciona un significativo crecimiento de la producción porcina, de alrededor del $112 \%$ en la primera década del siglo XXI. Dicho crecimiento se vio acompañado de un mayor desarrollo tecnológico en las explotaciones, como fue la implementación de la IA en el $85 \%$ de las hembras en sistemas en confinamiento, con la utilización anual de más de un millón de dosis seminales (Williams, 2015, citado por Arisnabarreta \& Allende, 2017).

Actualmente, en nuestro país, la obtención de los eyaculados y el procesamiento del material seminal se realizan mayoritariamente en los mismos establecimientos en que se encuentran las hembras (granjas comerciales) o en lugares muy próximos a estos. Una alternativa a esta estrategia son los centros de IA porcina, cuyo radio de acción regional asegura la conservación de la viabilidad y fertilidad del material seminal procesado.

\section{Parámetros indicativos de calidad seminal}

Cuando se conserva material seminal para realizar IA es importante tener en cuenta la calidad del semen utilizado, sobre la base de parámetros mínimos de aceptación que determinan si el eyaculado obtenido puede ser utilizado con éxito.

La evaluación del semen comprende el examen macroscópico, que incluye la medición del volumen, el $\mathrm{pH}$ mediante tiras reactivas y la 
Tabla 1. Valores de referencia y límites de aceptabilidad de diferentes parámetros de semen fresco porcino

\begin{tabular}{lcc}
\hline Parámetros & Valores de referencia & Límite de aceptabilidad \\
\hline Motilidad & $>75 \%$ & $60 \%$ \\
Concentración (fracción rica) & $700-800 \times 106 / \mathrm{ml}$ & \\
Malformaciones & $10-15 \%$ & $70 \%$ \\
$\begin{array}{l}\text { Espermatozoides normales } \\
\text { Acrosomas dañados }\end{array}$ & $10 \%$ & $75 \%$ \\
Acrosomas normales & & \\
$\mathrm{pH}$ & $6,8-7,3$ & \\
\hline
\end{tabular}

Extraido de Williams (2015)

apreciación del olor, el color y el aspecto. Por otro lado, en el examen microscópico se evalúa la motilidad individual, el recuento celular (concentración), las formas anormales, la vitalidad y el grado de aglutinación de la muestra. Otras evaluaciones complementarias que ayudan a categorizar la calidad seminal son el test de resistencia osmótica (ORT, por sus siglas en inglés), la evaluación de la integridad acrosomal y la identificación de gérmenes y antibiograma (Williams, 2015) (Tabla 1).

La evaluación del material seminal es de suma importancia debido a que es un indicador directo de la fertilidad de los verracos (Gadea et al., 1998) y debe tenerse en cuenta a la hora de seleccionar reproductores o decidir la continuidad de los mismos en el plantel (Tabla 2).

Finalmente, considerando que las cerdas son inseminadas con dosis seminales que contienen una determinada cantidad de espermatozoides y que son conservadas a la temperatura adecuada, la calidad de las mismas debe evaluarse para reducir el margen de error previo a la IA. Para ello se tendrán en cuenta los parámetros y valores considerados en el examen microscópico del semen fresco.

\section{Lugar de deposición de la dosis}

Según el lugar del útero en donde se deposita el material seminal, existen diferentes técnicas de IA en las que varían: el volumen, la cantidad de espermatozoides que se utilizan por dosis (concentración), y la longitud del dispositivo empleado para inseminar (catéter de inseminación). En la actualidad existen tres técnicas utilizadas en la especie porcina; de ellas, dos son muy sencillas de realizar y se utilizan rutinariamente en granjas comerciales. La primera de las técnicas desarrolladas fue la IA convencional (IAC), en la cual, la dosis seminal se deposita en el cuello del útero, ya sea en los anillos más caudales o en el tercio medio del cérvix, dependiendo del catéter que se utilice. La segunda fue la IA post- cervical (IAPC), que como su nombre lo indica, consiste en atravesar el cérvix y colocar la dosis en el cuerpo del útero. La tercera se denomina IA profunda (IAP) y, en este caso, la dosis seminal se deposita en uno de los cuernos uterinos, favoreciendo la aproximación de los espermatozoides a la unión útero-tubárica, lugar más cercano al sitio donde se produce la fecundación de los ovocitos. Esta técnica se reserva para utilizar semen descongelado, debido a que el mismo posee una sobrevida post-descongelación inferior al refrigerado a $15{ }^{\circ} \mathrm{C}$.

Actualmente, los verracos pueden producir entre 20 y 40 dosis de $2,5-3$ mil millones de espermatozoides diluidos en un volumen de $75^{-}$ $100 \mathrm{ml}$ para IAC, o bien, 40-60 dosis de 1,5 a 2 mil millones de espermatozoides en volúmenes menores para una IAPC. Esto demuestra que, con la sola modificación de la técnica de IA, se puede duplicar el aprovechamiento de los verracos, práctica ampliamente justificada en el caso de animales de alto índice genético (Knox, 2016). Estudios realizados para probar la eficiencia de la IA con baja cantidad de espermatozoides han dado resultados aceptables, tanto utilizando la técnica de IAC con dosis de 2 y 3 mil millones de espermatozoides en volúmenes convencionales (100 ml) (Feitsma, 2009; Olesen \& Hansen, 2009; Watson \& Behan, 2002) como realizando una IAPC con mil millones de espermatozoides en volúmenes reducidos (40$50 \mathrm{ml}$ ) (Vázquez et al., 2008) (Tabla 3).

\subsection{Inseminación artificial convencional}

Es la técnica más utilizada, simple y fácil de realizar, en la cual el semen diluido, con un volumen final de 70 a $100 \mathrm{ml}$, se coloca en el cérvix utilizando un catéter de IA de $54 \mathrm{~cm}$ de longitud (Arisnabarreta \& Allende, 2017).

La cantidad de espermatozoides probados para una única IAC ha oscilado entre 1 y $40 \mathrm{mil}$ millones de espermatozoides (Kemp \& Soede, 1997). Mientras que los primeros trabajos indicaron una fertilidad óptima con 5 a 10 mil millones 
Tabla 2. Valores seminales (media $\pm \mathrm{DE}$ ) de motilidad, morfoanomalías, gotas citoplasmáticas y colas en látigo y su relación con el porcentaje de parición de hembras inseminadas con semen de padrillos de baja, media y alta fertilidad

\begin{tabular}{lccccc}
\hline $\begin{array}{c}\text { Tipo de } \\
\text { fertilidad }\end{array}$ & $\begin{array}{c}\text { Parición } \\
\mathbf{( \% )}\end{array}$ & $\begin{array}{c}\text { Motilidad } \\
\mathbf{( \% )}\end{array}$ & $\begin{array}{c}\text { Morfoanomalías } \\
\mathbf{( \% )}\end{array}$ & $\begin{array}{c}\text { Gotas } \\
\text { citoplasmáticas } \\
\mathbf{( \% )}\end{array}$ & $\begin{array}{c}\text { Colas en } \\
\text { látigo } \\
\mathbf{( \% )}\end{array}$ \\
\hline Baja & $8 \pm 3,26$ & $63,7 \pm 3,1$ & $43 \pm 6,8$ & $26,8 \pm 5,6$ & $15,2 \pm 2,1$ \\
Media & $52,63 \pm 2,27$ & $74,5 \pm 1,7$ & $22,7 \pm 4$ & $14,2 \pm 3,4$ & $7,2 \pm 1,5$ \\
Alta & $89,03 \pm 1,82$ & $76,6 \pm 1$ & $11,1 \pm 1,9$ & $6,1 \pm 1,2$ & $4 \pm 1,2$ \\
\hline
\end{tabular}

Extraido de Williams (2015)

de espermatozoides, con los avances en los diluyentes, la calidad seminal y los materiales para la inseminación, la cantidad de espermatozoides se ha reducido gradualmente. Es así que en Europa se utilizan dosis de 1,5 a 2 mil millones y en otros países de 2,5 a 4 mil millones de espermatozoides (Kemp \& Soede, 1997), obteniendo escasos beneficios para la fertilidad al incrementar la cantidad de los mismos. Se ha mostrado poco efecto sobre la fertilidad cuando se inseminó con 1,3 o 6 mil millones de espermatozoides, siempre que la inseminación se realice entre 12 y 24 horas antes de la ovulación (Steverink et al., 1997). Actualmente, en muchas partes del mundo se realiza la IAC utilizando dosis de entre 2,5 y 3 mil millones de espermatozoides (Broekhuijse et al., 2011; Knox et al., 2008). Sin embargo, se han realizado ensayos a campo que sugieren que a medida que la cantidad de espermatozoides en la dosis aumentó de 2,5 a 4,5 mil millones también lo hizo el tamaño de la camada (Reicks \& Levis, 2008). Asimismo, otro estudio similar demostró que 3 mil millones de espermatozoides utilizados en una IAC aumentaron el tamaño de la camada y la tasa de parto en comparación con el uso de 2 mil millones de espermatozoides (Alm et al., 2006). Los resultados combinados de estos estudios sugieren que una mayor cantidad de espermatozoides proporciona algún tipo de resguardo contra los problemas relacionados con la fertilidad del semen, la estación del año, el operario y el momento de la IA. En la práctica, esta técnica usualmente utiliza una cantidad de espermatozoides por dosis de 3 mil millones (Hormaechea, 2016).

\subsection{Inseminación artificial post-cervical}

En la inseminación post o transcervical, el material seminal diluido en un volumen final de 30 a $50 \mathrm{ml}$, conteniendo 1,5 millones de células espermáticas por dosis, se deposita por vía no quirúrgica en el cuerpo del útero, por medio de una cánula que sobresale $15 \mathrm{a} 20 \mathrm{~cm}$ del interior de un catéter de IAC. La longitud del complejo catéter-cánula es de $73 \mathrm{~cm}$.

Estudios de campo han resultado en tasas de fertilidad similares al realizar dos IAPC, tanto con dosis de 1,5 como con 3 mil millones de espermatozoides en volúmenes reducidos (40-60 $\mathrm{ml}$ ). Por su parte, Hernández-Caravaca et al. (2012), utilizando dosis de mil millones de espermatozoides, lograron en promedio una tasa de partos del $84 \%$ y un tamaño de la camada de 13,9 lechones nacidos totales, valores más que aceptables para estos parámetros productivos. Adicionalmente, se ha comprobado que la IAPC en sí misma no mejora la fertilidad cuando se usa una mayor cantidad de espermatozoides (3 mil millones) (Peltoniemi et al., 2009). Si bien, Rozeboom et al. (2004) han reportado menor tasa de partos y tamaño de camada con menos de mil millones de espermatozoides, Mezalira et al. (2005) consiguieron una buena fertilidad utilizando solo 500 millones si la inseminación se realiza 24 horas antes de la ovulación.

Parece poco probable que los resultados de fertilidad sean similares para ambas técnicas de inseminación, dadas las diferencias que poseen, pero esto es posible gracias a que los elementos críticos importantes para la fertilidad se adaptan a cada tipo de inseminación, como son la cantidad de espermatozoides y el volumen de la dosis.

Con la finalidad de reducir la cantidad de espermatozoides por dosis, el uso comercial de la IAPC continúa aumentando en todo el mundo; cada vez más proveedores de reproductores o de material genético en dosis seminales, producen dosis con baja cantidad de espermatozoides en volúmenes reducidos y recipientes más pequeños.

A diferencia de la monta natural, en la IA no existe un tapón gelatinoso que obture el cérvix para disminuir el reflujo del material seminal luego del servicio (Lovell \& Getty, 1968). Esto predispone a que las contracciones miometriales que ayudan al transporte del esperma a través del cuerpo y cuernos uterinos, ocasionen el reflujo de parte de la dosis luego de la IA. Esta respuesta uterina se produce con el fin de eliminar el exceso de esperma y secreciones, preparando al útero para la futura preñez (Steverink et al., 1998). Está comprobado que, dentro de las 2 horas posteriores a la IA, el $60 \%$ de los espermatozoides de la dosis, son eliminados por polimorfonucleares neutrófilos 
Tabla 3. Comparación de la fertilidad y fecundidad de cerdas después de haber sido inseminadas dos veces en el mismo estro con un dispositivo estándar (Goldenpig®: control) y uno novedoso para su utilización en inseminación post-cervical (Deepgoldenpigig)

\begin{tabular}{lccccc}
\hline Dispositivo & $\begin{array}{l}\text { Dosis inseminante } \\
\text { (mil millones) }\end{array}$ & $\begin{array}{l}\text { Preñez } \\
(\%)\end{array}$ & $\begin{array}{l}\text { Parto } \\
(\%)\end{array}$ & Nacidos totales & Nacidos vivos \\
\hline & 1 & 66,2 & 65,8 & 10,3 & 9,0 \\
Goldenpig@ & 2 & 91,1 & 91,8 & 12,6 & 10,9 \\
& 3 & 91,3 & 91,1 & 12,5 & 10,9 \\
& & & & & \\
DeepgoldenpigTM & 1 & 88,7 & 86,9 & 12,1 & 10,9 \\
& 2 & 92,6 & 92,5 & 12,3 & 10,8 \\
Total $(\mathrm{N})$ & 3 & 91,8 & 90,5 & 12,3 & 11,0 \\
\hline
\end{tabular}

Extraido y modificado de Watson \& Behan (2002)

del endometrio y por el reflujo vaginal (RodríguezMartínez et al., 2005; Willenburg et al., 2003). Se ha demostrado que esto ocurre tanto al inseminar con diluyente solo, como utilizando una dosis seminal, aunque en este último caso, la presencia de espermatozoides pareció incrementar notablemente la respuesta celular (Rozeboom et al., 1998). Además, se observó que con la exposición al padrillo durante la IAC dicho reflujo disminuía (Willemburg et al., 2003).

Con la IAPC en muchos casos ocurren fugas o reflujos de dosis similares a los de la IAC, aunque esto es más común en la IAC por la incorrecta colocación del catéter y por la excesiva velocidad a la cual se deposita el semen. Asimismo, es normal que haya reflujo de dosis, cuando estas son voluminosas hasta 1-2 horas luego de la inseminación (Hernández-Caravaca et al., 2012).

Por otro lado, se dispone de pocos estudios sobre el uso de la IAPC en cerdas nulíparas. En condiciones de campo, y utilizando el dispositivo tradicional para cerdas multíparas, da Rosa Ulguim et al. (2018) observaron que la cánula solo llegaba $10 \mathrm{~cm}$ más allá del cuello uterino en el $44 \%$ de las cerdas nulíparas en el primer intento. En otro informe, se logró un éxito total en aproximadamente el $20 \%$ de los casos con un dispositivo para multíparas, aunque este bajo índice de éxito aumentó al 60,3\% en el primer intento con un dispositivo diseñado específicamente para la IAPC en primerizas (HernándezCaravaca et al., 2017). Resulta necesario realizar más investigaciones para optimizar la IAPC en hembras nulíparas, dado que existen diferencias en la porción craneal del cuello uterino entre nulíparas y multíparas (García-Vázquez et al., 2019). El diseño de dispositivos especiales de IA de acuerdo con la forma del cuello uterino puede ser una solución para mejorar el éxito de la IAPC en las diferentes categorías de cerdas (Llamas-López et al., 2019).

\subsection{Inseminación artificial profunda}

Finalmente, con el fin de reducir aún más la cantidad de espermatozoides en la dosis seminal, se desarrolló la IAP (Vázquez et al., 2005). Fue diseñada para el uso de semen criopreservado o sexado, con valores de viabilidad y fertilidad inferiores a los de semen fresco o refrigerado a $15{ }^{\circ} \mathrm{C}$, con el objetivo de incrementar al máximo la posibilidad de una preñez. En la IAP los espermatozoides se depositan en uno de los cuernos, lo más cerca posible de la unión útero-tubárica, con un catéter y una cánula de aproximadamente $148 \mathrm{~cm}$. Con esta técnica se minimiza el reflujo y los efectos de las células de la inflamación, particularmente polimorfonucleares neutrófilos, lo que permite reducir la cantidad de espermatozoides y el volumen de las dosis. Mediante esta técnica, se han logrado preñeces usando dosis de 50 a 200 millones de espermatozoides de semen fresco, de 70 a 140 millones de espermatozoides de semen sexado mediante citometría de flujo y de mil millones de espermatozoides congelados-descongelados (Roca et al., 2003).

Sin embargo, el alto costo del catéter para este procedimiento y las dificultades en la ejecución de la técnica, aún representan impedimentos para su implementación en granjas comerciales. Además, los resultados de la eficiencia reproductiva son inferiores cuando se los compara con los obtenidos con la IAPC (Tabla 4).

\section{Momento de la inseminación}

Si bien son varios los factores que influyen en la tasa de fecundación, como por ejemplo el entrenamiento de los operarios que realizan la detección de celo y la IA, la alimentación del plantel reproductor y factores medio ambientales, la calidad seminal junto con el momento de la 
Tabla 4. Tasas de fecundación y parto, y tamaño de camada de cerdas tratadas hormonalmente al destete e inseminadas una vez con procedimiento intrauterino profundo, usando un bajo número de espermatozoides frescos $\left(150 \times 10^{6}\right)$ y descongelados $\left(1000 \times 10^{6}\right) \mathrm{o}$ inseminadas convencionalmente utilizando semen descongelado $\left(6000 \times 10^{6}\right)$

\begin{tabular}{|c|c|c|c|}
\hline & \multicolumn{2}{|c|}{ Inseminación intrauterina profunda } & \multirow{2}{*}{$\begin{array}{l}\text { Inseminación convencional } \\
\text { Semen descongelado }\end{array}$} \\
\hline & Semen descongelado & Semen fresco & \\
\hline $\mathrm{N}^{\circ}$ cerdas inseminadas & 49 & 29 & 33 \\
\hline $\mathrm{N}^{\circ}$ cerdas que no retornaron al estro (\%) & $42 / 49(85,71)$ & $24 / 29(82,76)$ & $27 / 33(81,82)$ \\
\hline $\mathrm{N}^{\circ}$ cerdas preñadas al día $28(\%)$ & $39 / 49(79,59)$ & $24 / 29(82.76)$ & $26 / 33(78,79)$ \\
\hline $\mathrm{N}^{\circ}$ cerdas que abortaron $(\%)$ & $1 / 39(2,56)$ & $0(0,0)$ & $1(3,85)$ \\
\hline $\mathrm{N}^{\circ}$ cerdas al parto $(\%)$ & $38 / 49(77,55)$ & $24 / 29(82.76)$ & $25 / 33(75,76)$ \\
\hline Lechones nacidos (media \pm ES) & $354(9,31 \pm 0,41)$ & $239(9,96 \pm 0,32)$ & $240(9,6 \pm 0,53)$ \\
\hline
\end{tabular}

Extraido y modificado de Roca et al. (2003)

inseminación son factores determinantes para la ocurrencia de una preñez.

La IA puede realizarse en el mismo momento en el que se detecta el celo o bien pueden identificarse las hembras en celo para realizarla posteriormente. La detección de celo y la técnica de inseminación suelen estar relacionadas con el diseño de las granjas (animales confinados en jaulas o en corrales grupales acordes con las normas de bienestar animal). Kemp et al. (2005) comprobaron que la detección del celo puede realizarse sin dificultades independientemente del sistema de alojamiento en el que se encuentren los animales. Sin embargo, se observó que el grado de expresión del celo puede disminuir por efecto del reagrupamiento luego del destete (Rault et al., 2014) o por estar las cerdas en continuo contacto con los verracos (Knox et al., 2004). Por otro lado, la proximidad del verraco puede acentuar la expresión del celo e inducir la ovulación en cerdas primíparas y multíparas luego del destete, al trasladarlas al sitio de la granja donde toman contacto con el mismo, diariamente y de forma intermitente (sitio 1) (Langendijk et al., 2006).

Para las cerdas alojadas en jaula, la exposición al verraco se lleva a cabo durante 1 a 2 minutos mientras este recorre las hileras frente a las cerdas y simultáneamente un operario aplica presión en los cuartos traseros de estas.

En alojamientos grupales, la detección de celo se puede realizar observando el comportamiento de las cerdas cuando un macho es puesto en las cercanías del corral o dentro del mismo.

Una tercera alternativa para gestaciones grupales, aunque más costosa, es la detección de celo electrónica, que puede ayudar a identificar cerdas en celo usando registros de visitas a un macho adyacente, tomados durante 24 horas (Bressers et al., 1991; Cornou, 2006).

Considerando que aproximadamente entre el $75 \%$ y el $80 \%$ de las hembras del plantel reproductor son cerdas, y el resto son hembras de reposición, las cuotas de servicio se organizan sobre la base de un evento constante como es el destete. La mayoría de las cerdas expresan celo entre los días 4 y 6 post-destete y los registros indican que el $90 \%$ de las mismas muestran celo y se inseminan dentro de los primeros 7 días (Olesen, 2011, citado por Knox 2016; Yeste et al., 2014). Aunque la mayoría de las cerdas recibirán dos dosis seminales por ciclo, un bajo porcentaje de las mismas puede recibir una dosis o hasta tres dosis, según la duración del celo. En granjas que no disponen de un centro de IA dentro del establecimiento, las entregas programadas de dosis deberían ocurrir antes de que las cerdas comiencen a expresar el celo post-destete, manteniéndolas almacenadas a $15-17^{\circ} \mathrm{C}$.

Estos protocolos consideran la presencia del verraco durante la IA. Dicho manejo tiene por objetivo mantener a la cerda de pie y promover las contracciones miometriales que ayudan a la progresión de la dosis seminal a lo largo del útero (Langendijk et al., 2005). Sin embargo, se ha demostrado que la realización de la inseminación en ausencia del macho no tiene efectos perjudiciales sobre la progresión de la dosis, la retención de espermatozoides o la fertilidad (Willenburg et al., 2003), aunque su presencia tiene efectos facilitadores para la realización de la IA en primerizas.

Si bien actualmente la práctica de IA en presencia del macho es el procedimiento de rutina, requiere trabajo adicional para su movimiento y control. La técnica de IAPC, sin embargo, no requiere la presencia del padrillo en el momento de la inseminación, para no estimular las contracciones del útero (Langendijk et al., 2005).

En granjas comerciales se utiliza un sistema de inseminación múltiple, en el que las hembras reciben una dosis seminal por cada día 
Tabla 5. Modelo de protocolos utilizados para realizar inseminación artificial teniendo en cuenta la relación del intervalo destete-celo, en granjas donde se realizan dos detecciones diarias

\begin{tabular}{|c|c|c|c|c|}
\hline $\begin{array}{l}\begin{array}{l}\text { Presentación de } \\
\text { post-destete }\end{array} \\
\end{array}$ & Detección de celo & Día 1 de detección & Día 2 de detección & Día 3 de detección \\
\hline \multirow{2}{*}{$\begin{array}{l}\text { Temprano } \\
\text { (3-4 días post-destete) }\end{array}$} & matutina & celo & celo $^{\text {ra }}$ IA & sí hay celo $3^{\text {ra }}$ IA \\
\hline & vespertina & celo & celo $2^{\text {da }}$ IA & \\
\hline \multirow{2}{*}{$\begin{array}{l}\text { Normal } \\
\text { (5-7 días post-destete) }\end{array}$} & matutina & celo & celo $2^{\mathrm{da}}$ IA & sí hay celo $3^{\text {ra }}$ IA \\
\hline & vespertina & celo $1^{\text {ra }}$ IA & & \\
\hline \multirow{2}{*}{$\begin{array}{l}\text { Tardio } \\
\text { (>7 días post-destete) }\end{array}$} & matutina & celo $1^{\text {ra }} \mathrm{IA}$ & \multirow[t]{2}{*}{ sí hay celo $3^{\text {ra }}$ IA } & \\
\hline & vespertina & celo $2^{\text {da }}$ IA & & \\
\hline
\end{tabular}

que se detecta celo positivo (Diehl et al., 1984). La detección precisa del inicio del estro es importante, porque gran parte de los datos de fecundidad demuestran que proporcionar una única dosis de semen es menos efectivo que un sistema de IA múltiple. Como resultado, detectar celo diariamente ayuda a asegurar que un alto porcentaje de cerdas reciba inseminaciones múltiples (Flowers \& Esbenshade, 1993; Lamberson \& Safranski, 2000).

El grado de respuesta al reflejo de quietud de monta cambia al progresar el estro. Esto podría usarse para ajustar el momento óptimo para realizar la IA, debido a que dicha respuesta, ante la sola presencia del hombre (sin verraco), ocurre solo hacia la mitad del estro y más cerca del momento de ovulación (Langendijk et al., 2000). La duración del estro es variable y su importancia radica en la cantidad de inseminaciones que recibirá la cerda en relación al momento de la ovulación (Weitze et al., 1994). El estro dura de 45 a 65 horas y la ovulación se produce siempre en el último tercio (Soede \& Kemp, 1997). La mayoría de los estudios indican que la dehiscencia de todos los folículos ocurre en un lapso de 1 a 3 horas (Flowers \& Esbenshade, 1993; Soede et al., 1992; Soede et al., 1997).

Como la mayoría de las hembras del plantel reproductor son cerdas destetadas, la importancia del intervalo destete-estro y estroovulación determina el momento de la IA y la fertilidad del servicio (Kemp \& Soede, 1997).

Las cerdas que presentan celo poco después del destete tienen un estro de mayor duración, por lo que el intervalo inicio del celoovulación también se prolonga, en contraposición a cerdas que tienen su celo post-destete más tardío (celos e intervalo inicio del celo-ovulación más cortos). Es por esta relación que las hembras que manifiestan celo poco después del destete tienen más probabilidades de recibir una tercera dosis, mientras que las que lo hacen más tardíamente, generalmente reciben una sola IA. Sin embargo, en muchas granjas comerciales se realiza un ajuste del protocolo de IA según la manifestación de celo post-destete, retrasando el primer servicio en cerdas detectadas en celo en los días 3 y 4 postdestete, ya que la mayoría de estas permanecerá en celo durante 2 días más, y aun así recibirán dos dosis por ciclo (Tabla 5 ).

La fertilidad con el uso de la IA está muy influenciada por el intervalo entre la inseminación y la ovulación y explica gran parte de la variación en la tasa de preñez y el tamaño de la camada. El intervalo entre la inseminación y la ovulación puede influir en la viabilidad de los espermatozoides del reservorio oviductal (Soede et al., 1995), la fertilización del ovocito y la supervivencia embrionaria (Hunter, 1967; 1990).

Soede et al. (1995) evaluaron la tasa de fertilidad y la cantidad de embriones normales en cerdas inseminadas entre las 48 horas previas y las 16 horas posteriores a la ovulación (Tabla 6). Asimismo, Nissen et al. (1997) obtuvieron mayor tasa de parto y tamaño de la camada en cerdas inseminadas entre 24 horas previas y 4 horas posteriores a la ovulación. En todos los casos se comprobó que las inseminaciones realizadas demasiado temprano o demasiado tarde en el estro, y su relación con la ovulación, pueden reducir la fertilidad, e incluso si se realizan hacia finales del estro pueden aumentar la pérdida de embriones y causar endometritis (Kemp \& Soede, 1997).

\subsection{Reservorio espermático}

La formación del reservorio espermático después de la IA es un aspecto crítico para la fertilidad de los eyaculados. Solo una pequeña proporción de espermatozoides de la dosis seminal sobrevive al proceso de eliminación por reflujo y a los leucocitos endometriales, estableciéndose en 
Tabla 6. Estado de los embriones recuperados y de los ovocitos, por cada cerda y para cada intervalo de tiempo inseminación-ovulación de 8 horas $(n=151)$

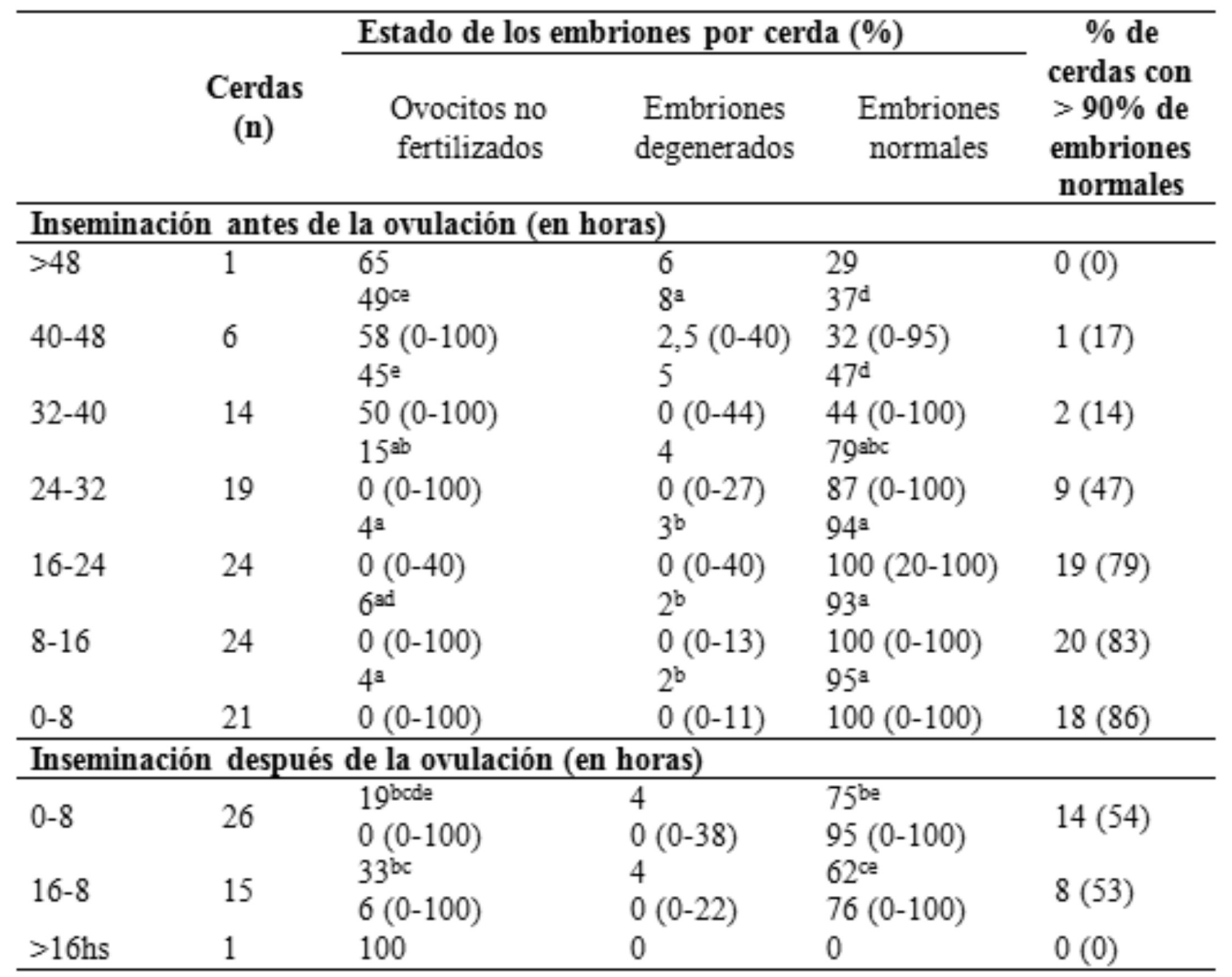

La mediana y el rango se expresan entre paréntesis. a b c e Los valores con diferentes letras en una columna difieren significativamente $(\mathrm{P}<0,05)$. Cerdas que fueron inseminadas más de 48 horas antes de la ovulación y más de 16 horas después de la ovulación no se incluyen en los análisis. Extraido y modificado de Soede et al, (1995).

reservorios en el oviducto (Claus et al., 1985b; First et al., 1968; Rodríguez-Martínez et al., 2005; Woelders \& Matthijs, 2001). Una escasa cantidad de espermatozoides llega al oviducto dentro de los 15 minutos posteriores a la IA; dicha cantidad se incrementa considerablemente luego de 2 horas. Colectivamente, estos espermatozoides conforman el reservorio espermático en las trompas, permaneciendo viables durante 24 horas (RodríguezMartínez et al., 2005). A pesar de encontrarse en el lugar donde ocurre la fertilización luego de unos minutos posteriores a la IA, los espermatozoides requieren de 2 a 4 horas de exposición al bicarbonato oviductal para modificar su motilidad y estructura de membrana y así capacitarse (Woelders \& Matthijs, 2001). La ubicación de los espermatozoides en el reservorio oviductal parece importante para su selección, capacitación, transporte y escape de la fagocitosis (Hunter, 1981; Rodríguez-Martínez et al., 2005). Dicho reservorio funciona para asegurar que los espermatozoides se mantengan en diferentes estadios de capacitación de manera de permitir la fertilización de los ovocitos a medida que llegan al oviducto (Rodríguez-Martínez et al., 2001). Este estado de capacitación diferencial también puede servir para modular la liberación de espermatozoides del reservorio, contribuyendo a evitar la polispermia (Rodríguez-Martínez et al., 2005).

\subsection{Factores que modifican la fertilidad de la dosis seminal}

En el eyaculado del verraco, más precisamente en el plasma seminal, existen hormonas y sustancias que influyen en la fertilidad espermática al modular la supervivencia y el transporte de los espermatozoides (Claus et al., 1985a). El agregado de plasma seminal, estrógenos o prostaglandinas (que se encuentran en el plasma 
seminal) u oxitocina (liberada por el útero) a una dosis de IA podría influir en las contracciones uterinas y en el transporte de semen (Flowers \& Esbenshade, 1993; Kirkwood et al., 2008; Weiler \& Claus, 1990). Además, se ha demostrado que el plasma seminal es importante para modular la respuesta inmune del útero después del apareamiento (Rodríguez-Mártinez et al., 2009) permitiendo la supervivencia de los espermatozoides antes de que se produzca la fagocitosis por parte de los polimorfonucleares del sistema inmune de la cerda (Rodríguez-Martínez et al., 2005).

Finalmente, la adición de oxitocina y prostaglandina F2a a una dosis de IA, tuvo efectos positivos, más claramente evidenciados cuando la fertilidad de la misma se vio comprometida por un factor limitante, como por ejemplo el tiempo de conservación de la dosis, la cantidad de espermatozoides/dosis o la estación del año (Maes et al., 2003; Peña et al., 1998).

\section{Conclusiones}

1. La inseminación artificial en la especie porcina está en constante desarrollo y permite el aprovechamiento de verracos genética-mente superiores y la aplicación de otras técnicas, como la congelación de semen, el sexado de espermatozoides y la IA a tiempo fijo.

2. El lugar de deposición de la dosis, ya sea de forma convencional, post-cervical o profunda, permite modificar la cantidad de espermatozoides y el volumen de la dosis, logrando un mayor aprovechamiento del potencial de los verracos. Cuanto más se aproxima el lugar de deposición a la unión útero-tubárica (zona de fertilización), menor cantidad de espermatozoides y menor volumen de la dosis seminal es requerida.

3. Cuando la IA se realiza a celo detectado, es importante instaurar protocolos que aseguren la aplicación de la dosis seminal y la formación de un adecuado reservorio de esperma-tozoides, lo más cercano posible al momento de la ovulación, para alcanzar los porcentajes de preñez y tamaños de camadas acordes a las exigencias de la especie.

Estos conocimientos constituyen la base para futuras mejoras de la técnica de IA en la especie porcina, para incrementar la fertilidad y, consecuentemente, el porcentaje de preñez y el tamaño de camada, mejorando así la eficiencia reproductiva y productiva de los establecimientos comerciales.

\section{Agradecimientos}

El presente trabajo fue financiado a través del Proyecto de Incentivos 11/V230 "Monitoreo de la salud animal y utilización de biotecnologías reproductivas para la mejora de la eficiencia reproductiva en animales de interés productivo y de compañía" bajo la dirección del Dr. R. Luzbel de la Sota y la co-dirección de las Dras. Sara Williams y María Alejandra Stornelli.

\section{Conflicto de intereses}

Todos los autores declaran que no existe conflicto de intereses, incluyendo entre estos últimos las relaciones financieras, personales o de otro tipo con otras personas $u$ organizaciones que pudieran influir de manera inapropiada en el trabajo.

\section{Bibliografía}

Alm K, Peltoniemi OAT, Koskinen E, Andersson M. 2006. Porcine field fertility with two different insemination doses and the effect of sperm morphology. Reproduction in Domestic Animals. 41(3):210-3.

doi: 10.1111/j.1439-0531.2005.00670.x

Arisnabarreta ER, Allende RA. 2017. Manual de inseminación artificial en porcinos. [libro electrónico] Buenos Aires: Ediciones INTA. Disponible en: http://www.revistataurus.com.ar/ uploads/productos/

20170903161417_manual_de_inseminacion_artifi cial_en_porcinos__arisnabarreta__allende_.pdf . [Consultado 28/11/2019]

Brassley P. 2007. Cutting across nature? The history of artificial insemination in pigs in the United Kingdom. Studies in History and Philosophy of Biological and Biomedical Sciences. 38(2):442-61.

doi: 10.1016/j.shpsc.2007.03.008

Bressers HPM, Te Brake JHA, Noordhuizen JPTM. 1991. Oestrus detection in group-housed sows by analysis of data on visits to the boar. Applied Animal Behaviour Science. 31(3-4):18393 .

\section{doi: 10.1016/0168-1591(91)90004-H}

Broekhuijse MLWJ, Feitsma H, Gadella BM. 2011. Field data analysis of boar semen quality. Reproduction in Domestic Animals. 46:59-63. doi: 10.1111/j.1439-0531.2011.01861.x

Cano A, García Mata E. Cría de cerdos. En: Cano A., García Mata E. 1953. Enciclopedia Agropecuaria Argentina. Buenos Aires, Editorial Sudamericana, pp: 71-121.

Claus R, Schopper D, Wagner HG, Weiler U. 1985a. Photoperiodic influences on reproduction of domestic boars, I. Light influences on testicular steroids in peripheral blood plasma and seminal plasma. Zentralblatt für Veterinärmedizin Reihe A. 
32:86-98.

doi: 10.1111/j.1439-0442.1985.tbo1920.x

Claus R, Weiler U, Wagner HG. 1985b. Photoperiodic influences on reproduction of domestic boars, II. Light influences on semen characteristics and libido. Zentralblatt für Veterinärmedizin Reihe A. 32:99-109.

doi: 10.1111/j.1439-0442.1985.tbo1921.x

Colenbrander B, Feitsma H, Grooten HJ. 1993. Optimizing semen production for artificial insemination in swine. Journal of Reproduction and Fertility. 48:207-15.

Cornou C. 2006. Automated oestrus detection methods in group housed sows: Review of the current methods and perspectives for development. Livestock Science. 105(1-3):1-11. doi: 10.1016/j.livsci.2006.05.023

Crabo B. 1991. Preservation of boar semen: a worldwide perspective. Proceedings, 2nd International Conference on Boar semen preservation, Beltsville MD, 1990, USDA, pp. 312.

da Rosa Ulguim R, Vier CM, Betiolo F, Sbardella PE, Bernardi ML, Wentz I, Mellagi AP, Bortolozzo FP. 2018. Insertion of an intrauterine catheter for post-cervical artificial insemination in gilts: a case report. Semina: Ciências Agrárias. 39(6):2833-88. doi: 10.5433/1679-0359.2018v39n6p2833

Diehl JR, Day BN, Stevermer EJ. 1984. Artificial insemination in swine. Pig industry handbook North Carolina Cooperative Extension Service. pp. 3-6.

Feitsma H. 2009. Artificial insemination in pigs, research and developments in the Netherlands, a review. Acta Scientiae Veterinariae. 37(1):61-71.

First NL, Short RE, Peters JB, Stratman FW. 1968. Transport and loss of boar spermatozoa in the reproductive tract of the sow. Journal of Animal Science. 27(4):1037-40.

doi: 10.2527/jas1968.2741037x

Flowers WL, Esbenshade KL. 1993. Optimizing management of natural and artificial matings in swine. Journal of Reproduction and Fertility. 48:217-28.

Franco R. 2012. Buenas prácticas de manejo en las distintas etapas productivas. En: Beyli ME, Brunori J, Campagna D, Cottura G, Crespo D, Denegri D, Ducommun ML, Faner C, Figueroa ME, Franco R, Giovannini F, Goenaga P, Lomello F, Lloveras M, Millares P, Odetto S, Panichelli D, Pietrantonio J, Rodríguez Fazzone M, Suárez R, Spiner N, Zielinsky G. 2012. Buenas prácticas pecuarias (BPP) para la producción y comercialización porcina familiar, Organización de las Naciones Unidas para la Agricultura y la Alimentación. pp. 141-54.

Gadea J, Matás C, Lucas, X. 1998. Prediction of porcine semen fertility by homologous in vitro penetration (hIVP) assay. Animal Reproduction Science. 54(2):95-108.

doi: 10.1016/So378-4320(98)oo144-4

García-Vázquez FA, Llamas-López PJ, Jacome MA, Sarrias-Gil L, Albors OL. 2019. Morphological changes in the porcine cervix: A comparison between nulliparous and multiparous sows with regard to post-cervical artificial insemination. Theriogenology. 127:120-9.

doi: 10.1016/j.theriogenology.2019.01.004

Hernández-Caravaca I, Izquierdo-Rico MJ, Matás C, Carvajal JA, Vieira L, Abril D, Soriano-Úbeda C, García-Vázquez FA. 2012. Reproductive performance and backflow study in cervical and postcervical artificial insemination in sows. Animal Reproduction Science. 136(1-2):14-22.

doi: 10.1016/j.anireprosci.2012.10.007

Hernández-Caravaca I, Llamas-López PJ, Izquierdo-Rico MJ, Soriano-Úbeda C, Matás C, Gardón JC, García-Vázquez FA. 2017. Optimization of post-cervical artificial insemination in gilts: Effect of cervical relaxation procedures and catheter type. Theriogenology. 90:147-52.

doi: 10.1016/j.theriogenology.2016.11.027

Hormaechea S. 2016. Inseminación artificial postcervical en cerdas. Tesina de pregrado para la orientación Producción Animal, Facultad de Ciencias Veterinarias, Universidad Nacional del Centro de la Provincia de Buenos Aires.

http://www.ridaa.unicen.edu.ar/xmlui/handle/ $123456789 / 1190$

Hunter RHF. 1967. The effects of delayed insemination on fertilization and early cleavage in the pig. Reproduction. 13(1):133-47.

doi: 10.1530/jrf.0.0130133

Hunter RHF. 1981. Sperm transport and reservoirs in the pig oviduct in relation to the time of ovulation. Reproduction. 63(1):109-17.

doi: 10.1530/jrf.0.0630109

Hunter RHF. 1990. Fertilization of pig eggs in vivo and in vitro. Journal of Reproduction and Fertility. 40: 211-26.

Kemp B, Soede NM. 1997. Consequences of variation in interval from insemination to ovulation on fertilization in pigs. Journal of Reproduction and Fertility. 52:79-89. 
Kemp B, Soede NM, Langendijk P. 2005. Effects of boar contact and housing conditions on estrus expression in sows. Theriogenology. 63(2):643-56. doi: 10.1016/j.theriogenology.2004.09.038

Kirkwood RN, Vadnais ML, Abad M. 2008. Practical application of seminal plasma. Theriogenology. 70(8): 1364-7.

doi: 10.1016/j.theriogenology.2008.07.018

Knox RV. 2016. Artificial insemination in pigs today. Theriogenology. 85(1):83-93.

doi: 10.1016/j.theriogenology.2015.07.009

Knox RV, Breen SM, Willenburg KL, Roth S, Miller GM, Ruggiero KM, Rodríguez-Zas SL. 2004. Effect of housing system and boar exposure on estrus expression in weaned sows. Journal of Animal Science. 82(10):3088-93.

doi: $10.2527 / 2004.82103088 \mathrm{x}$

Knox R, Levis D, Safranski T, Singleton W. 2008. An update on North American boar stud practices. Theriogenology. 70(8):1202-8.

doi: 10.1016/j.theriogenology.2008.06.036

Lamberson WR, Safranski TJ. 2000. A model for economic comparison of swine insemination programs. Theriogenology. 54(5):799-807.

doi: 10.1016/Soo93-691X(0o)oo391-5

Langendijk P, Soede NM, Bouwman EG, Kemp B. 2000. Responsiveness to boar stimuli and change in vulvar reddening in relation to ovulation in weaned sows. Journal of Animal Science. 78(12): 3019-26. doi: 10.2527/2000.78123019x

Langendijk P, Soede NM, Kemp B. 2005. Uterine activity, sperm transport, and the role of boar stimuli around insemination in sows. Theriogenology. 63(2):500-13.

doi: 10.1016/j.theriogenology.2004.09.027

Langendijk P, Soede NM, Kemp B. 2006. Effects of boar stimuli on the follicular phase and on oestrous behaviour in sows. Society of Reproduction and Fertility. 62:219-30.

Llamas-López PJ, López-Úbeda R, López G, Antinoja E, García-Vázquez FA. 2019. A new device for deep cervical artificial insemination in gilts reduces the number of sperm per dose without impairing final reproductive performance. Journal of Animal Science and Biotechnology. 10(1):11. doi: 10.1186/s40104-019-0313-1

Lovell JW, Getty R. 1968. Fate of semen in the uterus of the sow: histologic study of endometrium during the 27 hours after natural service. American Journal of Veterinary Research. 29(3): 609-25.
Maes DGD, Mateusen B, Rijsselaere T, De Vliegher S, Van Soom A, de Kruif A. 2003. Motility characteristics of boar spermatozoa after addition of prostaglandin F2 $\alpha$. Theriogenology. 6o(8):143543. doi: 10.1016/Soo93-691X(03)oo132-8

Marotta E. 1973. Extracción de semen en el cerdo: técnica y material. Revista de Medicina Veterinaria. 54:25-34.

Marotta E. 1978. Inseminación artificial en la especie porcina. Revista de Medicina Veterinaria. 59:310-2.

Mezalira A, Dallanora D, Bernardi ML, Wentz I, Bortolozzo FP. 2005. Influence of sperm cell dose and post insemination backflow on reproductive performance of intrauterine inseminated sows. Reproduction in Domestic Animals. 40(1):1-5.

doi: 10.1111/j.1439-0531.2004.00524.x

Nissen AK, Soede NM, Hyttel P, Schmidt M, D'hoore L. 1997. The influence of time of insemination relative to time of ovulation on farrowing frequency and litter size in sows, as investigated by ultrasonography. Theriogenology. 47(8):1571-82.

doi: 10.1016/Soo93-691X(97)00162-3

Olesen AK, Hansen C. 2009. Intrauterine insemination of sows by using a two-chamber semen bag system. Proceeding of 8th International Conference on Pig Reproduction, Nottingham, UK, pp. 81-2.

Palma GA, Brem G. Biotecnología de la reproducción. En: Palma GA. 2001. Biotecnología de la Reproducción. Buenos Aires, Ediciones INTA. pp. 1-14.

Peltoniemi OAT, Alm K, Andersson M. 2009. Uterine insemination with a standard AI dose in a sow pool system. Reproduction in Domestic Animals. 44(3):414-8.

doi: 10.1111/j.1439-0531.2008.01094.X

Peña FJ, Domínguez JC, Carbajo M, Anel L, Alegre B. 1998. Treatment of swine summer infertility syndrome by means of oxytocin under field conditions. Theriogenology. 49(4):829-36.

doi: 10.1016/Soo93-691X(98)ooo32-6

PigCHAMP Benchmarking. 2012. Use data to benchmark your farms. https:// www.pigchamp.com/benchmarking [Consultado el 06/11/2013].

Rault JL, Morrison RS, Hansen CF, Hansen LU, Hemsworth PH. 2014. Effects of group housing after weaning on sow welfare and sexual behavior. Journal of Animal Science. 92(12):5683-92. doi: $10.2527 /$ jas.2014-8238 
Reed HCB. Artificial insemination. En: Cole DJA, Foxcroft GR. 1982. Control of pig reproduction. London, Butterworth Scientific. pp. 65-90.

Reicks DL, Levis DG. 2008. Fertility of semen used in commercial production and the impact of sperm numbers and bacterial counts. Theriogenology. 70(8):1377-9.

doi: 10.1016/j.theriogenology.2008.07.019

Riesenbeck A. 2011. Review on international trade with boar semen. Reproduction in Domestic Animals. 46:1-3.

doi: 10.1111/j.1439-0531.2011.01869.x

Rillo SM. 1982. Reproducción e inseminación artificial porcina. Barcelona, Editorial AEDOS.

Roca J, Carvajal G, Lucas X, Vázquez JM, Martínez EA. 2003. Fertility of weaned sows after deep intrauterine insemination with a reduced number of frozen-thawed spermatozoa. Theriogenology. 6o(1):77-87.

doi: 10.1016/Soo93-691X(02)01330-4

Rodríguez-Martínez H, Kvist U, Saravia F, Wallgren M, Johannisson A, Sanz L, Peña FJ, Martínez EA, Roca J, Vázquez JM, Calvete JJ. 2009. The physiological roles of the boar ejaculate. Control of Pig Reproduction VIII. 66:1-21.

Rodríguez-Martínez H, Saravia F, Wallgren M, Tienthai P, Johannisson A, Vázquez J, Martínez E, Roca J, Sanz L, Calvete JJ. 2005. Boar spermatozoa in the oviduct. Theriogenology. 63(2): 514-35.

doi: 10.1016/j.theriogenology.2004.09.028

Rodríguez-Martínez H, Tienthai P, Suzuki K, Funahashi H, Ekwall H, Johannisson A. 2001. Involvement of oviduct in sperm capacitation and oocyte development in pigs. Reproduction Cambridge Supplement. 58:129-45.

Rozeboom KJ, Reicks DL, Wilson ME. 2004. The reproductive performance and factors affecting onfarm application of low-dose intrauterine deposit of semen in sows. Journal of Animal Science. 82(7): 2164-8. doi: 10.2527/2004.8272164x

Rozeboom KJ, Troedsson MHT, Crabo BG. 1998. Characterization of uterine leukocyte infiltration in gilts after artificial insemination. Reproduction. 114(2):195-9. doi: 10.1530/jrf.0.1140195

Soede NM, Helmond FA, Schouten WGP, Kemp B. 1997. Oestrus, ovulation and peri-ovulatory hormone profiles in tethered and loose-housed sows. Animal Reproduction Science. 46(1-2):13348. doi: 10.1016/So378-4320(96)01609-0
Soede NM, Kemp B. 1997. Expression of oestrus and timing of ovulation in pigs. Journal of Reproduction and Fertility. 52: 91-103.

Soede NM, Noordhuizen JPTM, Kemp B. 1992. The duration of ovulation in pigs, studied by transrectal ultrasonography, is not related to early embryonic diversity. Theriogenology. 38(4):65366. doi: 10.1016/0093-691X(92)90028-P

Soede NM, Wetzels CCH, Zondag W, De Koning MAI, Kemp B. 1995. Effects of time of insemination relative to ovulation, as determined by ultrasonography, on fertilization rate and accessory sperm count in sows. Reproduction. 104(1):99-106. doi: 10.1530/jrf.0.1040099

Steverink DWB, Soede NM, Bouwman EG, Kemp B. 1997. Influence of insemination-ovulation interval and sperm cell dose on fertilization in sows. Reproduction. 111(2):165-71.

doi: 10.1530/jrf.o.1110165

Steverink DWB, Soede NM, Bouwman EG, Kemp B. 1998. Semen backflow after insemination and its effect on fertilisation results in sows. Animal Reproduction Science. 54(2):109-19.

doi: 10.1016/So378-4320(98)oo146-8

Vázquez JM, Martínez EA, Roca J, Gil MA, Parrilla I, Cuello C, Caravajal G, Lucas X, Vázquez JL. 2005. Improving the efficiency of sperm technologies in pigs: the value of deep intrauterine insemination. Theriogenology. 63(2):536-47.

doi: 10.1016/j.theriogenology.2004.09.043

Vázquez JM, Roca J, Gil MA, Cuello C, Parrilla I, Vazquez JL, Martínez EA. 2008. New developments in low-dose insemination technology. Theriogenology. 70(8):1216-24. doi: 10.1016/j.theriogenology.2008.06.013

Watson PF, Behan JR. 2002. Intrauterine insemination of sows with reduced sperm numbers: results of a commercially based field trial. Theriogenology. 57(6):1683-93.

doi: 10.1016/So093-691X(02)00648-9

Weiler U, Claus R. 1990. Endocrine aspects of testicular function, especially hormones in the seminal plasma and their fate in the female reproductive tract: testicular steroids and their relevance for male and female reproductive functions. En: Johnson LA, Rath D. 1990. Boar semen preservation II. Berlin, Paul Parey Scientific Publishers. pp. 41-61.

Weitze K. 2000. Infertilidade estacional no suino. 3rd Simposio Internacional de Inseminaçào Artificial em Suinos. Porto Alegre, Brasil, pp. 50. 
Weitze KF, Wagner-Rietschel H, Waberski D, Richter L, Krieter J. 1994. The onset of heat after weaning, heat duration, and ovulation as major factors in AI timing in sows. Reproduction in Domestic Animals. 29(5):433-43.

doi: 10.1111/j.1439-0531.1994.tboo590.x

Willenburg KL, Miller GM, Rodríguez-Zas SL, Knox RV. 2003. Effect of boar exposure at time of insemination on factors influencing fertility in gilts. Journal of Animal Science. 81(1):9-15. doi: $10.2527 / 2003.8119$

Williams S, Fernández V. Evaluación, procesamiento y conservación de semen.
Inseminación artificial. En: Williams S. 2015. Atlas de Reproducción Porcina. Ciudad Autónoma de Buenos Aires, Inter-médica, pp. 25-32.

Woelders H, Matthijs A. 2001. Phagocytosis of boar spermatozoa in vitro and in vivo. Reproduction (Cambridge, England) Supplement. 58:113-27.

Yeste M, Estrada E, Pinart E, Bonet S, Miró J, Rodríguez-Gil JE. 2014. The improving effect of reduced glutathione on boar sperm cryotolerance is related with the intrinsic ejaculate freezability. Cryobiology. 68(2):251-61.

doi: 10.1016/j.cryobiol.2014.02.004 